\title{
Eficiencia del reflejo vestíbulo ocular mediante la aplicación de la prueba Video Head Impulse Test, en estudiantes de primer año de las Escuelas de Fonoaudiología y de Tecnología Médica de la Universidad de Valparaíso
}

\author{
Efficiency of the vestibular ocular reflex using Video Head Impulse Test \\ in students from the schools of Fonoaudiología and Tecnología Médica \\ of the University of Valparaíso
}

\begin{abstract}
André Gómez L1, Antonella Bruna $\mathrm{C}^{2}$, Daniela Franzoy E², María Sofía Julio $\mathbf{R}^{2}$, Romina Olivares $\mathrm{M}^{2}$, Nicole Vicencio P².
\end{abstract}

\begin{abstract}
RESUMEN
Introducción: Video Head Impulse Test es una nueva tecnología dirigida a la evaluación de los canales semicirculares, específicamente a la eficiencia del reflejo vestíbulo ocular. Es un procedimiento rápido, no invasivo y bien tolerado por el paciente. En Chile se evidencia una falta de estudios relacionados a esta prueba.

objetivo: Analizar los resultados de la eficiencia del reflejo vestíbulo ocular de los canales semicirculares horizontales, obtenidos mediante la aplicación de la prueba Video Head Impulse Test en estudiantes de las Escuelas de Fonoaudiología y de Tecnología Médica de la Universidad de Valparaíso.

Material y método: Estudio cuantitativo, descriptivo, no experimental, transversal descriptivo con un tipo de muestreo no probabilístico de participante voluntario. A los 80 estudiantes que cumplieron con los criterios de inclusión se les aplicó la prueba Video Head Impulse Test.

Resultados: El promedio de la ganancia del canal semicircular horizontal derecho fue de 0,92 $\pm 0,07$ y el de izquierdo fue de 0,85 $\pm 0,07$. Además el promedio de porcentaje de asimetría fue de $10 \% \pm 7,45$.

Conclusión: La investigación entrega datos referenciales en relación a los valores de normalidad para la eficiencia del reflejo vestíbulo ocular en una muestra específica, los que servirán como base para la ejecución de estudios posteriores.
\end{abstract}

Palabras clave: Reflejo vestíbulo ocular, video head impulse test, canal semicircular, ganancia, asimetría.

\footnotetext{
Fonoaudiólogo, Departamento de Audiología, Universidad de Valparaíso.

2 Fonoaudiólogo, Universidad de Valparaíso.
} 


\section{ABSTRACT}

Introduction: Video Head Impulse Test is a new technology directed to the evaluation of the semicircular canals, specifically the vestibular ocular reflex efficiency. It is a rapid, noninvasive and well tolerated procedure. In Chile there is a lack of studies related to this evaluation.

Aim: Analyze the results of the efficiency of the vestibular ocular reflex of the horizontal semicircular canals, using Video Head Impulse Test in students from the schools of Fonoaudiología and Tecnología Médica of the University of Valparaíso.

Material and method: A quantitative, descriptive, non-experimental descriptive stu$d y$ with a type of non-probability sampling with voluntary participant. The 80 students who fulfill the inclusion criteria were evaluated with Video Head Impulse Test.

Results: The mean gain of the right horizontal semicircular canal was $0.92 \pm 0.07$ and for the left was $0.85 \pm 0.07$. In addition the mean percentage of asymmetry was $10 \% \pm 7.45$.

Conclusions: The investigation delivery referential data regarding normal values for the efficiency of the vestibular ocular reflex in a specific sample, which serve as the basis for the implementation of future studies.

Key words: Vestibulo ocular reflex, video head impulse test, semicircular canal, gain, asymmetry.

\section{INTRODUCCIÓN}

La función del equilibrio en el ser humano resulta de la integración neurosensorial central de tres sistemas: visual, propioceptivo y vestibular ${ }^{1}$. Este último, participa en la mantención del equilibrio estático y dinámico, ya que detecta si el organismo se está desplazando o no y su relación con respecto a la fuerza de la gravedad².

En relación a la anatomía del sistema vestibular, este está compuesto por dos órganos otolíticos y por tres canales semicirculares (CSC) ${ }^{3}$. Estos últimos son sensibles a aceleraciones angulares de la cabeza, agrupándose y actuando en tres pares funcionales que yacen en planos perpendiculares entre sí. Los canales semicirculares horizontales, izquierdo y derecho, están situados en un plano común y funcionan en conjunto ( $1^{\circ}$ par funcional), mientras que cada conducto vertical anterior está situado en el mismo plano que el conducto vertical posterior contralateral $\left(2^{\circ} \text { y } 3^{\circ} \text { par funcional }\right)^{4}$ (Figura 1$)^{5}$.

\section{Reflejo vestíbulo ocular}

El reflejo vestíbulo ocular o RVO consiste en "la compensación de movimientos cefálicos por parte de los músculos extrínsecos oculares, de tal forma que la mirada pueda permanecer fija en un objeto independiente de los movimientos de la cabeza, por bruscos y rápidos que éstos sean"6.

La propiedad de este reflejo es su eficiencia, la cual se define como la capacidad de "generar mo-

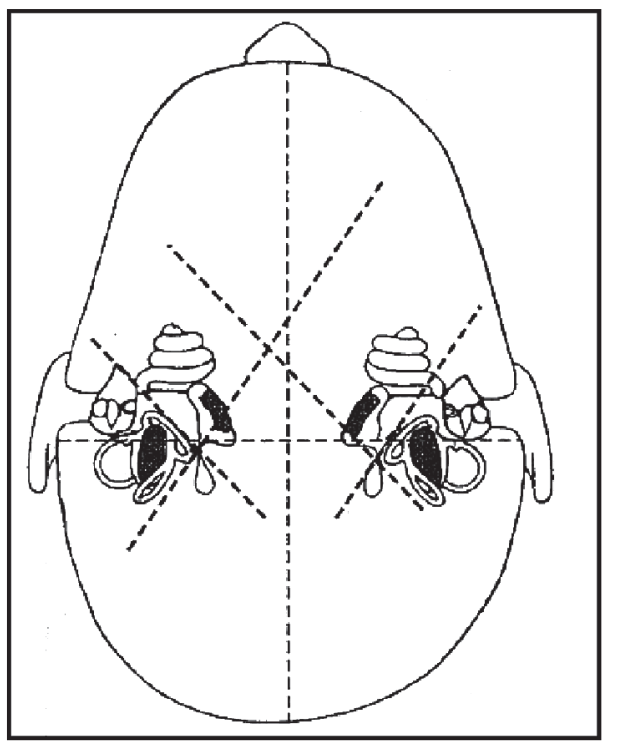

Figura 1. Esquema de orientación de los canales semicirculares (Extraído de Bartual, J., Pérez, N. "El sistema vestibular y sus alteraciones. Tomo I: Fundamentos y semiología”, 1999). 
vimientos oculares de fase lenta casi perfectamente compensatorios en dirección y velocidad para los movimientos de la cabeza"7. Asimismo, esta eficiencia tiene dos componentes siendo uno de ellos la ganancia, descrita como "Relación que existe entre el movimiento cefálico (input) y el desplazamiento de los ojos (output). Por lo tanto, la aceleración de los ojos es consignada como una función de la aceleración cefálica (ganancia RVO = aceleración ocular/aceleración cefálica) y su valor ideal en sujetos normales es considerado cercano a uno"7.

La asimetría entre las ganancias de los pares funcionales de los CSC es el otro componente de la eficiencia y en un paciente con lesión vestibular unilateral se definiría como "la relación existente entre la diferencia de la ganancia contralesional y la ganancia ipsilesional con la suma de ambas, multiplicada por cien". Sin embargo, otros autores señalan que la fórmula antes descrita presenta un comportamiento no lineal, entregando resultados menos precisos para indicar el porcentaje de asimetría vestibular. De este modo, estos autores presentan una fórmula con comportamiento lineal ${ }^{9}$. Un estudio realizado en el año 2008 en Australia, donde se evaluó a sujetos sanos a través de bobina de búsqueda escleral en campo magnético (BECM), concluye que es aceptable un valor no mayor a $24 \%$ $\pm 2,3^{8}$. Considerando estos componentes, es posible concluir que las respuestas del RVO serían un modo de evaluar la función de los CSC individualmente?

Cuando existe algún déficit en los CSC, se produce una alteración del RVO y de todas sus características. Físicamente, los ojos se mueven en la misma dirección de la rotación de la cabeza, perdiendo la estabilidad visual del objeto al que se mira y, como consecuencia, deben efectuar unos movimientos sacádicos correctivos (catch-up saccades) hacia el lado opuesto a la rotación. Existen dos tipos de sacadas correctivas, las descubiertas y las cubiertas. Las primeras se producen al final del movimiento cefálico y son fácilmente detectables por un evaluador capacitado. Las segundas surgen durante el movimiento cefálico y no pueden ser detectadas a ojo desnudo?

\section{Evaluación del reflejo vestíbulo ocular}

Con los avances entecnologías dirigidas al estudio de Ios CSC, se han creado exámenes complementarios a los empleados en la clínica actual. Uno de ellos es Head Impulse Test (HIT), que consiste en generar movimientos impredecibles, de pequeña amplitud, alta velocidad y gran aceleración sobre la cabeza del paciente, este estando a ojo desnudo ${ }^{7}$.

En 1963, Robinson diseñó una bobina de búsqueda escleral en campo magnético (BECM o scleral search-coil) que, hasta la fecha, sigue siendo considerada el gold standard para el registro de los movimientos tridimensionales oculares y cefálicos ${ }^{7}$. Esta técnica permite el registro del RVO durante los impulsos cefálicos, posibilitando el estudio del eje de rotación ocular, de las ganancias, de la asimetría y de las latencias desencadenadas por rotaciones cefálicas en los planos de los pares funcionales de Ios $\operatorname{CSC}^{10}$. Cabe destacar que, a pesar de su utilidad, es un método molesto para el paciente, caro, invasivo y de difícil implementación clínica ${ }^{7}$.

\section{Video Head Impulse Test}

Video Head Impulse Test (v-HIT) surge debido a la necesidad de crear un nuevo examen complementario que supla las desventajas de los anteriores. Éste se define como una herramienta objetiva que permite la evaluación de la eficiencia del RVO, detectando los movimientos oculares con un acelerómetro y una cámara de alta velocidad. Por lo tanto, logra registrar alteraciones sutiles del RVO, alcanzando una especificidad de $93 \%$ y una sensibilidad de $74 \%{ }^{11}$.

Esta prueba evalúa la respuesta de cada uno de los seis CSC ${ }^{12}$. Para su ejecución, el examinador, debe realizar sobre el paciente impulsos cefálicos con características similares a los de HIT, con velocidades entre $150 \%$ y $250 \% \mathrm{~s}^{10}$. En cada impulso, v-HIT registra tanto el movimiento de la cabeza como el del ojo, graficando dos curvas de velocidad angular en el tiempo y calculando un promedio de ganancia de los impulsos cefálicos realizados y un porcentaje de asimetría ${ }^{11}$.

Por lo tanto, como ventajas, v-HIT analiza y muestra la morfología de los dos tipos de movimientos sacádicos de refijación del RVO (sacadas descubiertas y cubiertas) ${ }^{13}$. Además, es un procedimiento rápido, no invasivo, bien tolerado y reproducible en el tiempo por distintos examinadores entrenados ${ }^{7}$. Más aún, los rangos de estimulación con impulsos cefálicos en v-HIT se asemejan más a los que el sujeto emplea en 
su vida cotidiana y, debido a su simpleza, permite la evaluación en casos de vértigo agudo, incluso, en un contexto de sala de urgencias. De esta manera, logra ser un complemento en el diagnóstico y seguimiento del paciente con patología vestibular ${ }^{11}$.

En Chile, se evidencia una falta de estudios específicos dedicados a v-HIT.

\section{OBJETIVO}

El objetivo de esta investigación fue analizar los resultados de la eficiencia del RVO de los canales semicirculares horizontales (CSCH), obtenidos mediante la aplicación de la prueba v-HIT, en estudiantes de primer año de las Escuelas de Fonoaudiología y de Tecnología Médica de la Universidad de Valparaíso. Mientras que los objetivos específicos fueron describir los valores promedio de ganancia del CSCH del oído derecho e izquierdo y describir los valores de asimetría entre las ganancias de ambos CSCH.

\section{MATERIAL Y MÉTODO}

Se realizó un estudio de tipo cuantitativo, con unalcance descriptivo y un diseño no experimental transversal descriptivo. El tipo de muestreo fue no probabilístico, por lo que la muestra quedó conformada por 80 estudiantes de primer año, matriculados en 2014 en la casa central de las Escuelas de Fonoaudiología o de Tecnología Médica de la Universidad de Valparaíso. La edad de los participantes fluctuó entre 18 a 25 años (con promedio de 19,15 años y desviación estandar de 1,42 años) dividiéndose en 13 hombres y 63 mujeres. Todos los participantes cumplieron con criterios de inclusión y exclusión que apuntaron a demostrar ausencia de patología auditiva y vestibular.

Para la selección de la muestra, a los estudiantes que firmaron el consentimiento informado se les realizó una anamnesis auditiva y vestibular, una otoscopía y una audiometría de barrido. Luego, a los estudiantes seleccionados se les realizó la prueba v-HIT con el instrumento e-HITUSB (casa comercial BioMed), donde los procedimientos fueron los siguientes: en primer lugar, el examinador le solicitaba al estudiante tomar asiento en una silla ubicada a un metro de la pared y luego le colocaba las gafas de video-oculografía. Todo lo anterior rea- lizado en el Laboratorio Vestibular de la carrera de Fonoaudiología de la Universidad de Valparaíso.

En segundo lugar, para calibrar el instrumento, el estudiante debía mirar sin mover su cabeza hacia tres puntos ubicados horizontalmente con una distancia de $36,4 \mathrm{~cm}$ entre ellos, siguiendo el orden: izquierda, centro y derecha.

En tercer lugar, para iniciar la evaluación, se le indicaba al estudiante que debía mantener su mirada fija en el punto central, con la cabeza inclinada $30^{\circ}$ aproximadamente, mientras que el investigador, ubicado detrás, le sujetaba la cabeza con las manos y le realizaba 10 movimientos, de manera rápida, brusca e impredecible, hacia la derecha e izquierda en un rango entre $15^{\circ}-25^{\circ}$ y con una velocidad sobre los $150^{\circ} /$ s.

Finalmente, los resultados obtenidos fueron consignados en una base de datos y procesados a través de tablas, gráficas y estadísticas, mediante el uso de los programas computacionales Excel 2007 y SPSS Statistics versión 19.

\section{RESULTADOS}

En relación a los resultados obtenidos de la investigación, se analizarán las dos variables del estudio: ganancia de cada canal semicircular horizontal (CSCH) y asimetría entre las ganancias.

En primer lugar, en cuanto a la ganancia del $\mathrm{CSCH}$ derecho, el histograma señala una distribución simétrica. Esto quiere decir, que los datos se distribuyen alrededor de la media y existe baja información en los extremos (Figura 2). Además según el test de normalidad de Kolmogorov-Smirnov (K-S), la probabilidad de significación es del 0,200, lo que implica que los datos obedecen a una distribución normal. Es importante señalar que el 52,5\% de la muestra que correspondió a 42 estudiantes presentó una ganancia entre 0,885 y 0,985.

En segundo lugar, en cuanto a la ganancia del $\mathrm{CSCH}$ izquierdo, se puede describir que en el histograma los datos se distribuyeron de forma normal y simétrica, al igual que los datos del CSCH derecho (Figura 3). Además el $67,5 \%$ de la muestra, que correspondió a 54 estudiantes, presentó una ganancia entre 0,755 y 0,895.

Comparando ambos resultados, la media de ganancia obtenida para el CSCH derecho alcanzó un valor de 0,92 con una desviación estándar de 0,07. Mientras 


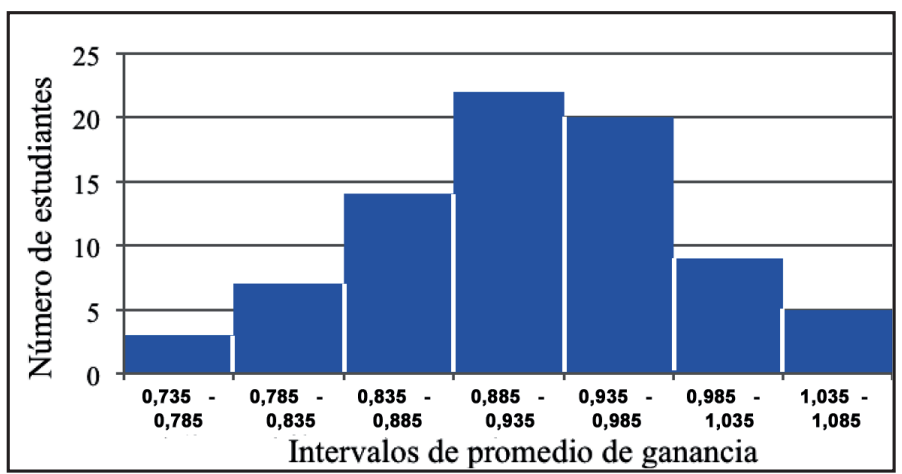

Figura 2. Distribución de los valores promedio de ganancia del canal semicircular horizontal derecho de los estudiantes de primer año de las Escuelas de Fonoaudiología y Tecnología Médica de la Universidad de Valparaíso.

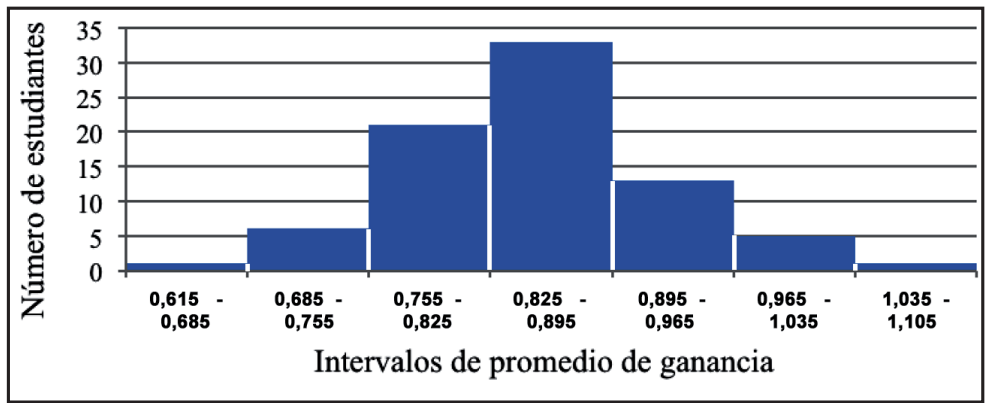

Figura 3. Distribución de los valores promedio de la ganancia del canal semicircular horizontal izquierdo de los estudiantes de primer año de las Escuelas de Fonoaudiología y Tecnología Médica de la Universidad de Valparaíso.

que para el CSCH izquierdo se obtuvo una media de ganancia de 0,85 con una desviación estándar de 0,07, al igual que en el oído derecho. Además, se puede destacar una diferencia de 0,07 entre las medias (Tabla 1).

En tercer lugar, con respecto a la segunda variable, asimetría entre las ganancias, se puede mencionar que los datos en el histograma se encuentran mayormente distribuidos hacia el lado izquierdo (Figura 4). Según el test de normalidad de K-S, la probabilidad de significación alcanza el 0,007, lo que implica que los datos no obedecen una distribución normal. Además, el 58,75\% de la muestra que correspondió a 47 estudiantes presentó una asimetría entre $2,5 \%$ y $14,5 \%$.
Finalmente, se debe mencionar que la media de la última variable mencionada alcanzó un valor de $10 \%$ con una desviación estándar de 7,45 (Tabla 2).

\section{DISCUSIÓN}

Antes de abordar las discusiones de la investigación, es importante recalcar, que ésta contó con una muestra de individuos jóvenes (edades menores a 25 años) lo que se traduce en que los resultados sólo pueden ser utilizados como referencia para una población con estas mismas características. Lo

Tabla 1. Medidas descriptivas de la ganancia de cada canal semicircular horizontal derecho e izquierdo

\begin{tabular}{|cccccccc|}
\hline CSCH & Media & Mediana & Moda & \multicolumn{2}{c}{ Cuartil } & D. estándar & Coef. De variación \\
& & & 1 & 3 & & \\
\hline Derecho & 0,92 & 0,92 & 0,94 & 0,88 & 0,97 & 0,07 & $7,64 \%$ \\
Izquierdo & 0,85 & 0,85 & 0,84 & 0,80 & 0,89 & 0,07 & $8,55 \%$ \\
\hline
\end{tabular}




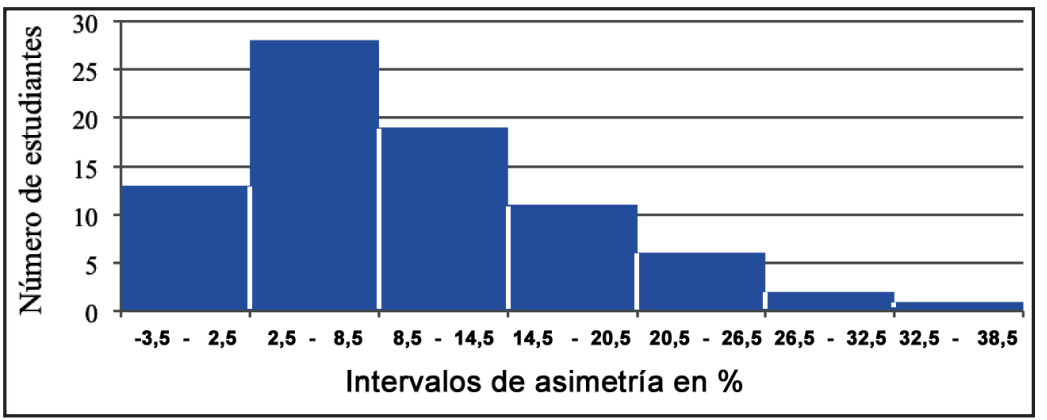

Figura 4. Distribución de la asimetría entre las ganancias de ambos canales semicirculares horizontales de los estudiantes de primer año de las Escuelas de Fonoaudiología y Tecnología Médica de la Universidad de Valparaíso.

Tabla 2. Medidas descriptivas de la asimetría de las ganancias de los canales semicirculares horizontales

\begin{tabular}{|c|ccccccc|}
\hline \multirow{3}{*}{ Asimetría } & Media & Mediana & Moda & \multicolumn{2}{c}{ Cuartil } & D. estándar & Coef. de variación \\
\cline { 2 - 7 } & 10 & 8 & 5 & 5 & 14,25 & 7,45 & $74,38 \%$ \\
\hline
\end{tabular}

anterior se ve respaldado por un trabajo reciente, que evidenció una disminución en la función del RVO de adultos mayores con edades entre $70 \mathrm{y}$ 95 años, mostrando resultados de la prueba HIT anormales en $50 \%$ de la muestra ${ }^{14}$. De esta forma, los resultados que se obtengan en la prueba HIT variarán de acuerdo al rango etario de los sujetos en estudio, principalmente en edades avanzadas.

En la literatura revisada, fue posible encontrar diversos estudios relacionados con los objetivos planteados, cuyos resultados fueron similares a los obtenidos en esta investigación. En un estudio, realizado en Australia en el año 2013, se analizaron las ganancias de cada CSC por separado, obteniendo para el CSCH derecho una ganancia de 1,03; y para el izquierdo, de $0,92^{15}$. En Surcorea, igualmente se obtuvieron valores de ganancia para el CSCH derecho de 0,92; y para el izquierdo, de $0,95^{16}$. En tanto, en un estudio español, se señala que los valores para la ganancia en el plano horizontal fueron de 0,94 $\pm 0,06$ para el CSCH derecho y de $1 \pm$ 0,07 para el izquierdo ${ }^{10}$. Por último, en otro estudio australiano, se obtuvieron valores de ganancia en el plano horizontal de 0,88 $\pm 0,05$ para el $\mathrm{CSCH}$ derecho y de 0,91 $\pm 0,12$ para el izquierdo $0^{17}$.

Cabe destacar que las medias de las ganancias de ambos CSCH $(0,92$ y 0,85$)$, obtenidas en la presente investigación, fueron desiguales, con una diferencia de 0,07 , donde la ganancia del CSCH izquierdo fue inferior a la del derecho. Esto último también se observa en los resultados obtenidos en el primer estudio mencionado, aunque la diferencia de las ganancias fue de $0,11^{15}$. En otra investigación, dentro de una muestra de sujetos sanos, se consideró el dato de ganancia más bajo para ambos $\operatorname{CSCH}(0,71$ y 0,66$)$, obteniendo, del mismo modo, una diferencia de 0,05. Además, en este último estudio, se postulan posibles factores que conducen a este suceso, explicando que se podría deber, por una parte, a las diferencias existentes entre las vías del RVO que llegan a los músculos extraoculares; y por otra parte, a la imparcialidad del evaluador que generó diferencias sistemáticas en los peak de aceleraciones a la derecha y a la izquierda ${ }^{18}$.

Un hecho importante, ocurrido durante el procedimiento de la aplicación de la prueba v-HIT a los estudiantes, fue la obtención de algunos valores de ganancia superiores a 1,00. Esta situación se menciona en un estudio del año 2013, donde se consideraron como normales, valores de ganancia del RVO de hasta 1,2. Los autores señalan que esto podría ocurrir cuando no existe un ajuste suficiente de las gafas 10 que provocaría un pequeño deslizamiento de ellas, que sería interpretado por la cámara como un desplazamiento de la posición de los ojos, lo que el sistema procesaría como un aumento artificial de la velocidad de éstos. Sin embargo, también indican que estos valores podrían producirse incluso con las gafas bien ajustadas, sin proponer otras posibles causas ${ }^{11}$. 
Con respecto a los valores de asimetría entre las ganancias, se obtuvo una media de 10 con una desviación estándar de 7,45. Además, con el test de normalidad de K-S, se comprobó que los datos están concentrados en los valores bajos (cercanos a cero) de la variable, lo que no implica un error en la medición 0 en el análisis, sino más bien, una asimetría más cercana al $0 \%$. En la literatura también se menciona que un porcentaje cercano a $0 \%$ señalaría resultados concordantes con sujetos sin patología vestibular periférica unilateral de base $e^{8,9}$.

Es importante no perder de vista que los valores de asimetría siempre deben ser mirados con cautela, ya que muchas veces valores considerados normales (simétricos) pueden enmascarar alteraciones del RVO, especialmente en disfunciones vestibulares bilaterales, que tienen una alta prevalencia en adultos mayores ${ }^{19}$. En estos casos la ganancia de cada CSC pudiese ser muy baja, sin embargo simétrica. De acuerdo a lo anterior se sugiere la observación individual de la ganancia de cada canal complementario (en nuestro estudio CSCH izquierdo y derecho) y la observación de sacadas correctivas que pudiesen indicar una disfunción del RVO.

En cuanto a los procedimientos aplicados en el estudio; a cada estudiante que cumplió con los criterios de inclusión se le realizaron 10 impulsos cefálicos pasivos, bruscos e impredecibles hacia la derecha e izquierda, respectivamente, en un rango entre $15^{\circ}-25^{\circ}$, con una velocidad sobre los $150^{\circ}$ s. Se optó por elegir un solo evaluador para toda la muestra y de esta manera eliminar la posible variabilidad de los resultados que se podían obtener entre distintos examinadores. Sin embargo esta elección podría ser a la vez una debilidad, ya que la asimetría generada por las ganancias de los $\mathrm{CSCH}$ izquierdo y derecho podrían ser explicadas por un sesgo del examinador en la técnica utilizada para realizar la prueba, más que a las características fisiológicas de la muestra. De esta forma surge de manera natural la necesidad de contar con estudios que evalúen la confiabilidad interexaminador de esta prueba y ver si existen diferencias significativas.

Los estudios mencionados anteriormente, obtuvieron valores de ganancia para cada CSCH y uno de ellos para la asimetría. Sin embargo, es necesario resaltar que todos siguieron diferentes procedimientos al realizar la evaluación. Por un lado, en el estudio de MacDougall, McGarvie, Halmagyi, Curthoys y Weber
(2013), fue empleada la prueba v-HIT simultáneamente con BECM. En éste, participaron 7 sujetos normales sentados a un metro de distancia del punto fijo y cuatro evaluadores distintos que les realizaron 20 impulsos cefálicos impredecibles en tiempo y dirección con una velocidad de $50 \%$ a $250 \% / \mathrm{s}^{15}$.

Por otro lado, las demás investigaciones revisadas evalúan diversos tamaños muestrales de sujetos sanos con el método de BECM, ejecutando rotaciones cefálicas pasivas e impredecibles de amplitudes entre $10^{\circ}$ y $20^{\circ}$ y velocidades entre $200 \%$ a $400 \%$ s. No obstante, aunque fue un instrumento diferente al empleado en el presente estudio, BECM es considerada el gold standard para poder determinar la eficiencia del RVO y, además, la prueba V-HIT fue validada en un estudio del año 2009, comparando sus resultados con los de BECM, donde se concluyó que ambos datos no fueron significativamente diferentes entre personas normales ${ }^{20}$. Por lo tanto, aunque las metodologías fueron diferentes, los datos obtenidos son similares.

Independiente de los valores obtenidos tanto en las ganancias de cada CSCH como en el de asimetría, es importante destacar la ausencia total de sacadas correctivas en la muestra. Este hallazgo cobra suma importancia al momento de realizar evaluaciones a pacientes jóvenes, donde la presencia de una sacada correctiva indicaría inequívocamente un estado patológico, ya que como se demostró en esta investigación, sujetos jóvenes, sanos y asintomáticos no debiesen presentar sacadas correctivas.

\section{CONCLUSIÓN}

Los valores obtenidos con la prueba v-HIT se asemejan a los propuestos en la literatura revisada. En relación a los datos de ganancia promedio de ambos CSCH éstos presentan una distribución normal.

La relevancia de este estudio es el uso de una nueva tecnología conocida como v-HIT, poco empleada en Chile. La investigación entrega datos referenciales en relación a los valores de normalidad para la eficiencia del RVO en una muestra específica de sujetos jóvenes.

La ausencia de sacadas correctivas en la muestra fue un hallazgo de valor clínico considerable, sugiriendo consecuentemente que la presencia de éstas en sujetos jóvenes, independiente de los 
valores de ganancia de los CSC y asimetría, se relacionan directamente con una disfunción del RVO.

Aunque los objetivos propuestos se cumplieron a cabalidad, la tensión cervical que presentaron algunos estudiantes provocó limitaciones en el grado y la velocidad de los giros cefálicos. Ante esta situación, se propone realizar masoterapia previa a la aplicación de la prueba v-HIT.

En definitiva v-HIT ha demostrado ser un examen sencillo, rápido y cómodo para el paciente que ayuda al diagnóstico y seguimiento de alteraciones vestibulares. Sin embargo, debido a la baja cantidad de estudios en Chile, aún persisten interrogantes en cuanto a los procedimientos e interpretación de los resultados. Por este motivo seguir esta línea de investigación facilitaría la utilización de esta prueba y aportaría a la otoneurología en Chile.

\section{BIBLIOGRAFÍA}

1. Visser J, Carpenter M, Van Der Kooij H, Bloem B. The clinical utility of posturography. J Clin Neurophysiol 2008; 119: 2424-36.

2. OLIVA M. La exploración oculomotora. Disponible en: http://webs.ono.com/nistagmoteca/OCULOMOT. pdf. Consultado el 24 de abril de 2014.

3. Hain T, Helminski J. Anatomy and Physiology of the Normal Vestibular System. En: Herdman S, Vestibular Rehabilitation. Philadelphia: F.A Davis Company, 2007; 2-18.

4. Martín A, Rubio M. Bases Neurofisiológicas del Equilibrio Postural. Disponible en: http:// gredos.usal.es/jspui/bitstream/10366/115263/1/ NeurofisiologiaEquilibrioPostural.AMMartin.pdf. Consultado el 3 de abril de 2014.

5. Bartual J, Pérez N, Barona $R$ y col. El sistema vestibular y sus alteraciones. Tomo I: Fundamentos y semiología. Barcelona: Masson, S.A 1999.

6. Breinbauer H, Anabalón J. Prueba de impulso cefálico. Rev Otorrinolaringol Cir Cabeza Cuello 2011; 71: 123-30.

7. Carriel C, Rojas M. Prueba de impulso cefálico: Bases fisiológicas y métodos de registro del reflejo vestíbulo oculomotor. Rev Otorrinolaringol Cir Cabeza Cuello 2013; 73: 206-12.
8. Weber K, Aw S, Todd M, McGarvie L, Curthoys I, Halmagyı G. Head Impulse Test in unilateral vestibular loss. Vestibulo-ocular reflex and catch-up saccades. J Neurol 2008; 70: 454-63.

9. Mathur N. Common: Vestibular cochlear Disorders I. India: Elsevier 2011.

10. Boleas-Aguirre M, Miglaccio A, Carey J. Registro del reflejo vestibulo oculomotor con la técnica de la bobina corneal en campo magnético (scleral search coil). Revisión de afecciones vestibulares periféricas. Acta Otorrinolaringol Esp 2007; 58: 321-6.

11. Breinbauer H, Anabalón J, Aracena K, Nazal D, Baeza M. Experiencia en el uso video-impulso cefálico (vHIT) en la evaluación del reflejo vestíbulo-ocular para el canal semicircular horizontal. Rev Otorrinolaringol Cir Cabeza Cuello 2013; 73: 115-24.

12. Magliulo G, Gagliardi S, Appiani M, Iannella G, GAGLIARDI M. Selective vestibular neurolabyrinthitis of the lateral and superior semicircular canal ampulla and ampullary nerve. Ann Otol Rhinol Laryngol 2012; 121: 640-4.

13. Blödow A, Pannasch S, Walther L. Detection of isolated covert saccades with the video head impulse test in peripheral vestibular disorders. Auris Nasus Larynx 2012; 40: 348-51.

14. Agrawal Y, Dávalos-Bichara M, Zúniga MG, CareY JP. Head Impulse Test Abnormalities and Influence on Gait Speed and Falls in Older Individuals. Otol Neurotol 2013; 34: 1729-35.

15. MacDougall H, McGarvie L, Halmagy G, Curthoys I, Weber K. The Video Head Impulse Test (vHIT) Detects Vertical Semicircular Canal Dysfunction. Journal PLOS ONE 2013; 8: 1-10.

16. KIm J, Jung H. Inferior vestibular neuritis. J Neurol 2012; 259: 1553-60.

17. Aw D, Halmagy G, Pohl D, Curthoys I, Yavor R, Todd M. The Effect of Unilateral Posterior Semicircular Canal Inactivation on the Human Vestibulo-Ocular Reflex. Acta Otolaryngol 1995; 115: 260-2.

18. Jorns-Häderli M, Straumann D, Palla A. Accuracy of the bedside Head Impulse Test in detecting vestibular hypofunction. J Neurol Neurosurg Psychiatry 2007; 78: 1113-8.

19. FIFE TD, BALOH RW. Disequilibrium of unknown cause in older people. Ann Neurol 1993; 34: 694-702.

20. Weber K, MacDougall H, Halmagy G, Curthoys I. Impulsive testing of semicircular-canal function using video-oculography. Ann N Y Acad Sci 2009; 1164: 486-91. 